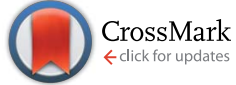

Cite this: J. Mater. Chem. A, 2015, 3, 1896

Received 9th November 2014 Accepted 15th December 2014

DOI: $10.1039 / c 4 t a 06058 j$

www.rsc.org/MaterialsA

\section{Conjugated microporous polymers with excellent electrochemical performance for lithium and sodium storage $\uparrow$}

\author{
Shengliang Zhang, $t^{\mathrm{ab}}$ Wei Huang, $\stackrel{t}{\mathrm{ab}}^{\mathrm{a}} \mathrm{Pu} \mathrm{Hu},{ }^{\text {ab }}$ Changshui Huang, ${ }^{\text {*a }}$ Chaoqun Shang, ${ }^{\text {ab }}$ \\ Chuanjian Zhang, ${ }^{*}$ Renqiang Yang ${ }^{\star a}$ and Guanglei Cui ${ }^{\star a}$
}

Conjugated microporous polymers, which exhibit high specific capacity, superior cycle stability and remarkable rate capability, are explored as high-performance electrode materials for lithium and sodium storage. Their excellent electrochemical performance can be attributed to their conductive frameworks, plentiful redox-active units, high specific surface area and homogeneous microporous structure.

In an effort to address the energy crisis and environmental issues, clean and sustainable energy systems have been investigated, such as solar cells, fuel cells and rechargeable batteries. Currently, lithium ion batteries (LIBs) dominate the portable consumer electronic market due to their high energy density. ${ }^{1,2}$ But because of the high cost and limited resources of lithium (Li), it is urgent to find a substitute for Li to meet the demands for rechargeable batteries. Recently, sodium ion batteries (SIBs) have been recognized as a low cost alternative to LIBs for next generation battery systems and large scale energy storage devices due to the natural abundance of sodium (Na) and the similar chemical properties of $\mathrm{Na}$ and $\mathrm{Li}^{3-5}$ However, the commonly used electrode materials in state-of-the-art LIBs and SIBs contain transitional metals, ${ }^{6-8}$ which are resource-limited and non-environmentally friendly. As an alternative, organic electrode materials have attracted more and more interest in recent years due to their low cost, high electrochemical performance, resource sustainability, environmental friendliness, structure diversity and flexibility. ${ }^{9-11}$ During the past decades, a large variety of organic electrode materials have been reported for capacitors ${ }^{11,12}$ and rechargeable batteries, such as conjugated carboxylates, ${ }^{13-15}$ conjugated polymers ${ }^{16,17}$ and

${ }^{a}$ Qingdao Institute of Bioenergy and Bioprocess Technology, Chinese Academy of Sciences, No. 189 Songling Road, 266101, Qingdao, China. E-mail: huangcs@ qibebt.ac.cn; yangrq@qibebt.ac.cn; cuigl@qibebt.ac.cn

${ }^{b}$ University of Chinese Academy of Sciences, No. 19A Yuquan Road, 100049, Beijing, China

$\dagger$ Electronic supplementary information (ESI) available. See DOI: 10.1039/c4ta06058j

\$ These authors contributed equally to this work. polynitroxide radicals, ${ }^{18}$ demonstrating the potential of organic electrodes for the next generation of green, sustainable and versatile energy storage devices.

In recent years, conjugated microporous polymers (CMPs), combining $\pi$-conjugated skeletons with permanent nanopores, have received increasing interest as an important branch of organic porous polymers. ${ }^{19,20}$ Compared with other inorganic or inorganic-organic hybrid porous materials, CMPs have intrinsic advantages such as a high degree of $\pi$-conjunction, homogeneous microporous structure, ultrahigh specific surface area, diversity and flexibility in the molecular design. Because of their unique features, CMPs have shown great potential in gas absorption, ${ }^{21,22}$ gas separation, ${ }^{23,24}$ heterogeneous catalysis ${ }^{25,26}$ and so on. However, there have been only a few reports of CMPs applied for Li or Na storage. Recently, Jiang and coworkers have applied CMPs for supercapacitors ${ }^{27}$ and cathodes of LIBs. $^{28}$ Sakaushi and co-workers applied bipolar porous polymeric frameworks for all-organic ${ }^{29}$ and $\mathrm{Na}-$ organic energy storage devices ${ }^{30}$ exhibiting excellent electrochemical performance. These results indicate that CMPs have enormous potential as green sustainable and flexible electrode materials for next generation energy storage devices.

In this work, we synthesize the polymer 4,7-dicarbazyl[2,1,3]-benzothiadiazole (PDCzBT) by $\mathrm{FeCl}_{3}$ oxidation coupling polymerization and investigate its electrochemical performance for Li and Na storage. The prepared PDCzBT shows an ultrahigh specific surface area that provides abundant active sites for storage reactions, and a uniform microporous structure that is beneficial for the rapid transport of electrons and ions. The skeleton of PDCzBT is built with plentiful redox-active units, which provide abundant energy storing modules. PDCzBT delivers an n-type reversible redox behavior with the reaction of $\mathrm{Li}^{+} / \mathrm{Na}^{+}$doping-dedoping into/from the polymer chains. The assembled batteries based on PDCzBT electrodes exhibit excellent electrochemical performance for $\mathrm{Li}$ and $\mathrm{Na}$ storage, including high specific capacity, outstanding cycle stability and superior rate performance. These results indicate that CMPs are 
promising electrode materials with high capacity, outstanding rate capability and long cycle life for next generation energy storage devices.

\section{Experimental}

\section{Sample preparation}

Tetrahydrofuran (THF) and chloroform were purified by distillation prior to use. 2,1,3-Benzothiadiazole (99\%), carbazole (96\%), cuprous oxide (96\%), ferric chloride (97\%), dimethylacetamide (DMAc, 99.8\%), and other solvents were purchased from J\&K or Aldrich and used as received.

Synthesis of 4,7-dicarbazyl-[2,1,3]-benzothiadiazole (DCzBT). To a mixture of 2,1,3-benzothiadiazole (1.470 g, $5.0 \mathrm{mmol}$ ), carbazole (2.508 g, $15.0 \mathrm{mmol})$, and cuprous oxide (2.862 g, 20.0 $\mathrm{mmol}$ ), $10 \mathrm{~mL}$ DMAc was added. The suspension was heated to $160{ }^{\circ} \mathrm{C}$ and refluxed for $24 \mathrm{~h}$ under nitrogen. After cooling to room temperature, the mixture was filtered to remove the cuprous oxide and then the filtrate was poured into water. The suspension was filtered and washed with water and ethanol to obtain a red brown solid. The final product DCzBT was purified by silica gel column chromatography (510 mg, 21\%). ${ }^{1} \mathrm{H}$ NMR $\left(600 \mathrm{MHz}, \mathrm{CDCl}_{3}\right): \delta(\mathrm{ppm})=8.22(\mathrm{~d}, 4 \mathrm{H}), 8.07(\mathrm{~s}, 2 \mathrm{H}), 7.45(\mathrm{~m}$, $4 \mathrm{H}), 7.37$ (m, 4H), 7.33 (d, 4H). ${ }^{13} \mathrm{C}$ NMR (150 MHz, $\left.\mathrm{CDCl}_{3}\right): \delta$ $(\mathrm{ppm})=152.64,141.02,129.72$, 127.75, 126.09, 124.09, 120.81, $120.58,110.43$.

Synthesis of polymer 4,7-dicarbazyl-[2,1,3]-benzothiadiazole (PDCzBT). The solution of monomer DCzBT (200 mg, 0.43 $\mathrm{mmol}$ ) dissolved in $30 \mathrm{~mL}$ of anhydrous chloroform was dropwise transferred to a suspension of ferric chloride (920 mg, 3.44 $\mathrm{mmol}$ ) in $20 \mathrm{~mL}$ of anhydrous chloroform. The solution mixture was stirred for $24 \mathrm{~h}$ at room temperature under nitrogen protection, and then $100 \mathrm{~mL}$ of methanol was added to the above reaction mixture. The resultant precipitate was collected by filtration and washed with methanol and concentrated hydrochloric acid solution. After being extracted using a Soxhlet extractor with methanol for $24 \mathrm{~h}$, and then with THF for another $24 \mathrm{~h}$ extraction, the desired polymer was collected and dried in a vacuum oven at $80{ }^{\circ} \mathrm{C}$ overnight. Brown powder; yield: $96 \%$. Anal. calcd for $\mathrm{C}_{30} \mathrm{H}_{18} \mathrm{~N}_{4} \mathrm{~S}$ : C, 77.23; N, 12.01; H, 3.89; S, 6.87. Found: C, 77.20; N, 12.12; H, 3.94; S, 6.62\%.

\section{Characterization}

Morphological information was obtained using a field emission scanning electron microscope (FESEM, HITACHI S-4800) and transmission electron microscope (TEM, HITACHI H-7650). ${ }^{1} \mathrm{H}$ and ${ }^{13} \mathrm{C}$ NMR spectra were recorded with a Bruker Avance III model $600 \mathrm{MHz}$ using $\mathrm{CDCl}_{3}$ as a solvent. ${ }^{13} \mathrm{C} \mathrm{CP} / \mathrm{MAS}$ solidstate NMR measurement was carried out on a Bruker Avance III model $400 \mathrm{MHz}$ NMR spectrometer at a MAS rate of $5 \mathrm{kHz}$. The FT-IR spectrum was collected in attenuated total reflection (ATR) mode on a Thermo Nicolet 6700 FT-IR Spectrometer. Thermogravimetric analysis (TGA) was carried out using a SDT Q600 (V20.9 Build 20) with a temperature ramp of $10{ }^{\circ} \mathrm{C} \mathrm{min}^{-1}$ from $20{ }^{\circ} \mathrm{C}$ to $800{ }^{\circ} \mathrm{C}$ under a $\mathrm{N}_{2}$ atmosphere. Nitrogen adsorption-desorption measurements were performed at $77 \mathrm{~K}$ using a Quantachrome Autosorb gas-sorption system.

\section{Electrochemical measurements}

The electrochemical experiments were performed in 2032 cointype cells. The working electrodes were prepared by mixing 60 wt $\%$ PDCzBT with 30 wt\% Super P and 10 wt $\%$ polytetrafluoroethylene (PTFE) binders. The obtained electrode samples were rolled into slices and cut into square pieces of $1 \mathrm{~cm} \times 1 \mathrm{~cm}$ (the areal loading of the total materials is about $3 \mathrm{mg} \mathrm{cm}^{-2}$ and the areal loading of the active materials is around $1.8 \mathrm{mg}$ $\mathrm{cm}^{-2}$ ), then pasted on a stainless steel current-collector under a pressure of $15 \mathrm{MPa}$, followed by drying in a vacuum oven at $120{ }^{\circ} \mathrm{C}$ for $4 \mathrm{~h}$ to be used as the working electrode. Pure Li (or $\mathrm{Na}$ ) foil was used as the counter electrode, which was separated from the working electrode by using a Celgard 2500 polymeric separator. The electrolyte was $1 \mathrm{M} \mathrm{LiPF}_{6}$ in ethylene carbonate (EC)-dimethyl carbonate (DMC)-diethyl carbonate (DEC) $(1: 1: 1, \mathrm{v} / \mathrm{v} / \mathrm{v})$ containing $5 \%$ (by volume) vinylene carbonate (VC) or $1 \mathrm{M} \mathrm{NaClO}_{4}$ in EC-DMC $(1: 1, \mathrm{v} / \mathrm{v})$ containing $2 \%$ (by volume) fluoroethylene carbonate (FEC). The cells were assembled in an argon-filled glovebox with the concentrations of moisture and oxygen less than $1 \mathrm{ppm}$. The galvanostatic charge-discharge cycling performance was measured using a LAND battery testing system. Cyclic voltammetry (CV) was performed using an IM6 electrochemical workstation at a scan rate of $0.2 \mathrm{mV} \mathrm{s}^{-1}$. The electronic conductivity of PDCzBT was carried out by a linear voltage scanning method (the detailed measuring method is shown in the ESI $\dagger$ ). The capacity was calculated based on the mass of PDCzBT.

\section{Results and discussion}

The polymer 4,7-dicarbazyl-[2,1,3]-benzothiadiazole (PDCzBT) was synthesized by $\mathrm{FeCl}_{3}$ oxidation coupling polymerization ${ }^{23,31}$ as shown in Scheme 1. The SEM image (Fig. 1a) shows that PDCzBT consists of relatively uniform sub-micron spheres. As can be seen in Fig. 1b, the TEM images indicate the porous structure of PDCzBT. The molecular structure of PDCzBT was assessed with ${ }^{13} \mathrm{C} \mathrm{CP} / \mathrm{MAS}$ solid nuclear magnetic resonance (NMR), as shown in Fig. 1c. The characteristic peak at 152 ppm orresponds to the carbon in the $-\mathrm{C}=\mathrm{N}$ groups. The peak at 141 ppm is ascribed to carbons connecting to the $\mathrm{N}$ atom in the carbazole groups. The signal at $110 \mathrm{ppm}$ and the broad peaks from 120 to $129 \mathrm{ppm}$ are assigned to the other carbon atoms of the aromatic rings in the polymers. A more detailed analysis of

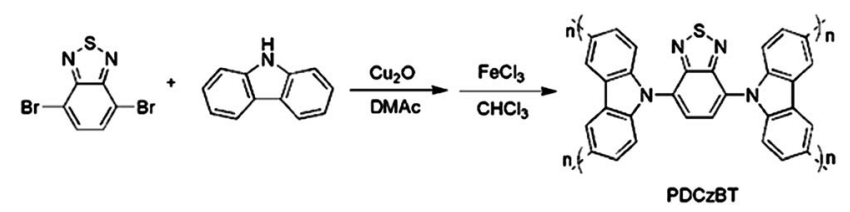

Scheme 1 Synthetic route of conjugated microporous polymer PDCzBT. 

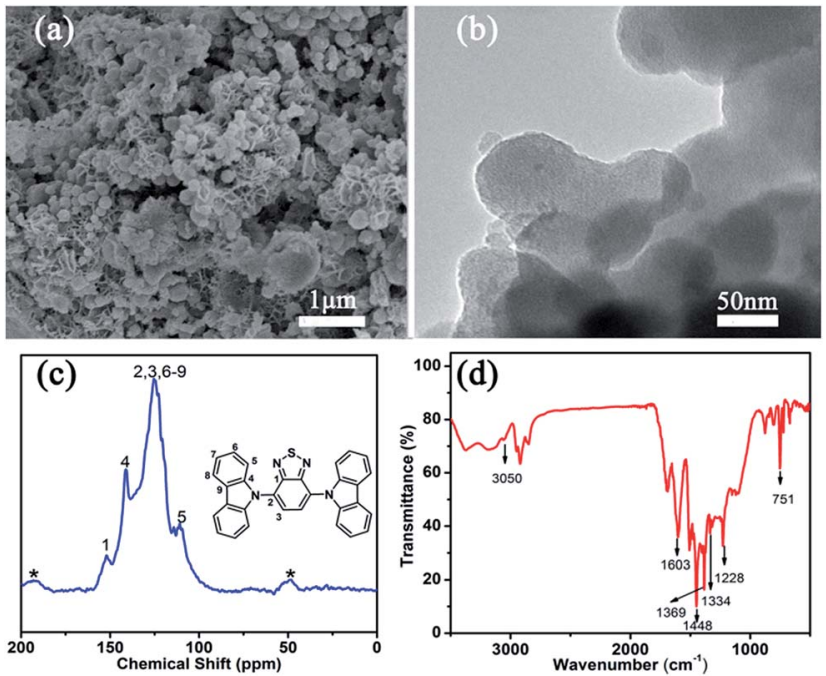

Fig. 1 (a) The SEM image, (b) TEM image, (c) ${ }^{13} \mathrm{C} C P / M A S$ solid NMR, and (d) FT-IR spectrum of PDCzBT.

the structure (monomer and polymer) was confirmed by the FTIR spectra (Fig. S1 $\uparrow$ and 1d). The spectra reveal the following absorption peaks: the bands around $751 \mathrm{~cm}^{-1}$ are due to ring deformations of the aromatic structure; the peaks at 1228, 1334, $1369 \mathrm{~cm}^{-1}$ may be the stretching frequencies of the structure such as the N-S-N, C-N and N-S moiety; the bands around $1448-1603 \mathrm{~cm}^{-1}$ should be the double bond $(\mathrm{C}=\mathrm{C}$ and $\mathrm{C}=\mathrm{N})$ stretching vibration in the structure; the peak around $3049 \mathrm{~cm}^{-1}$ is the stretching frequency of the $\mathrm{C}-\mathrm{H}$ bonds in the aromatic structure. The thermostability of PDCzBT was also investigated by thermogravimetric analysis (TGA) as it is crucial to the safety of rechargeable batteries. The resultant PDCzBT exhibited a high thermal stability without decomposing up to $350{ }^{\circ} \mathrm{C}$ (Fig. S2†).

The pore structure of the polymer was evaluated by a nitrogen adsorption-desorption isotherm at $77 \mathrm{~K}$. The curve depicted in Fig. 2a displays a type I isotherm with a rapid uptake at low relative pressure according to IUPAC classifications, ${ }^{32}$ which indicates the microporous nature of PDCzBT. There is a gradual rise and small hysteresis at relatively high pressure, consistent with the presence of interparticular voids, which could be ascribed to porosities existing between the highly aggregated nanoparticles. ${ }^{33}$ The calculated Brunauer-EmmettTeller (BET) specific surface area and the total pore volume $\left(V_{\text {tol }}\right)$
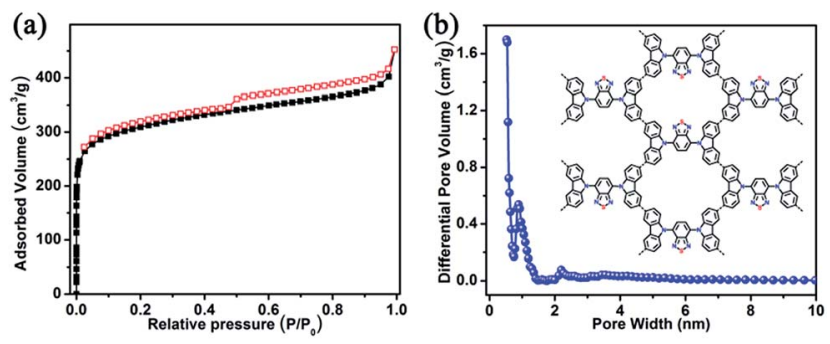

Fig. 2 (a) $\mathrm{N}_{2}$ adsorption-desorption isotherm and (b) pore-size distribution of PDCzBT (the inset is its structural representation). of PDCzBT are $1166 \mathrm{~m}^{2} \mathrm{~g}^{-1}$ and $0.7 \mathrm{~cm}^{3} \mathrm{~g}^{-1}$, respectively. The surface and pore volume are comparable to or higher than previously reported CMPs. ${ }^{27-30}$ The micropore volume $V_{0.1}$ is $0.45 \mathrm{~cm}^{3} \mathrm{~g}^{-1}$, and simultaneously the $V_{0.1} / V_{\text {tol }}$ value is 0.64 , which means that micropores are dominant in PDCzBT. This is also certified by the pore size distribution (Fig. 2b). It reveals Ithat PDCzBT has a dominant ultramicropore width at 0.52 and $0.86 \mathrm{~nm}$, indicating the relatively uniform micropores in the polymer. The inherent ultramicropore and large pore volume are accessible to $\mathrm{Li}$ and $\mathrm{Na}$ ion transport and its high surface area endows PDCzBT with abundant active sites for $\mathrm{Li}$ and $\mathrm{Na}$ storage.

\section{Electrochemical performance of PDCzBT for Li storage}

The electrochemical performance in terms of lithium storage was evaluated using 2032 coin-type half cells. As shown in the cyclic voltammogram (CV) profiles (Fig. 3a), during the first cathodic scan, an irreversible band appeared at 1.0-0.5 V, corresponding to the electrochemical decomposition of an electrolyte for the formation of a solid electrolyte interface (SEI) film on the electrode surface. The $\mathrm{CV}$ bands in a low potential region of 0-0.5 $\mathrm{V}$ emerged as a pair of asymmetric redox peaks with a larger cathodic branch than the anodic branch, reflecting the doping-dedoping reaction of $\mathrm{Li}^{+}$into/from the polymer chains. ${ }^{16,30}$ The reaction mechanism can be based on eqn (1). However, the initial coulombic efficiency is as low as $49.1 \%$ (Fig. S3†), which is ubiquitous in carbon-based electrodes especially in polymer electrodes.

$$
\mathrm{PDCzBT}+x \mathrm{Li}^{+}+x \mathrm{e}^{-} \leftrightarrow \mathrm{Li}_{x}{ }^{+} \mathrm{PDCzBT}^{x-}
$$
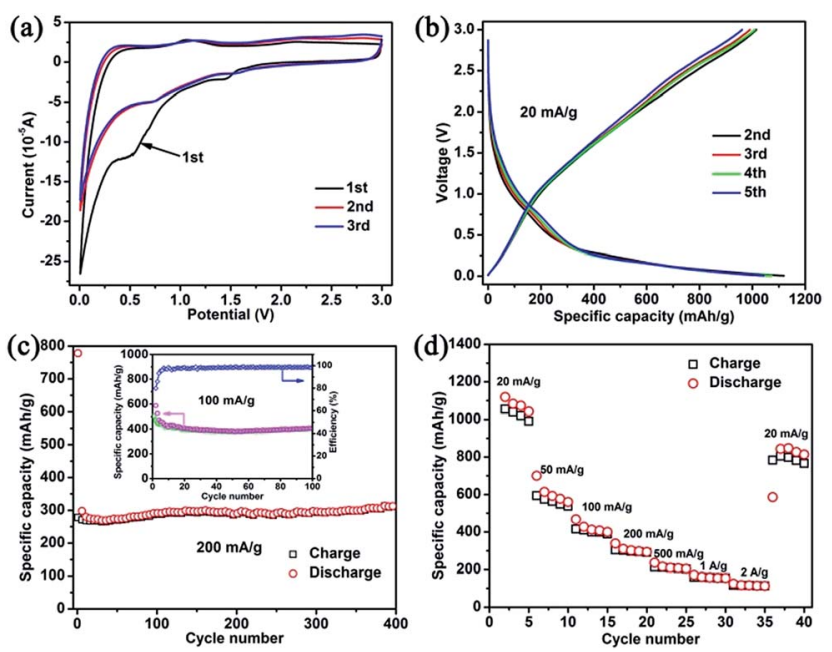

Fig. 3 Electrochemical performance of the PDCzBT electrode for LIBs. (a) Cyclic voltammogram (CV) profiles. (b) Galvanostatic chargedischarge profiles at a current density of $20 \mathrm{~mA} \mathrm{~g}^{-1}$. (c) Cycle performance at a current density of $200 \mathrm{~mA} \mathrm{~g}^{-1}$ and the inset is cycle performance and coulombic efficiency at $100 \mathrm{~mA} \mathrm{~g}^{-1}$. (d) Rate performance at varied current density ranging from 20 to $2000 \mathrm{~mA} \mathrm{~g}^{-1}$. 
Fig. 3b displays the typical charge-discharge curves of the coin cells cycled in the n-dopable potential region, which is consistent with the $\mathrm{CV}$ curves. The large part of specific capacity $(>70 \%)$ in the region below $0.5 \mathrm{~V}$ (Fig. S4 $\dagger$ ) corresponds to $\mathrm{Li}^{+}$ doping into the polymer chains. ${ }^{16,30}$ The specific capacity above $0.5 \mathrm{~V}$ might be attributed to the $\mathrm{Li}^{+}$absorbing on the surfaces/ interfaces of PDCzBT. ${ }^{34}$ The detailed Li storage mechanism can be further studied by the CVs (Fig. S5 $\dagger$ ) at different scan rates, ${ }^{35}$ which demonstrate that it is a mixed process involving both $\mathrm{Li}^{+}$ doping into the polymer chains and absorbing on the surfaces/ interfaces. It is worth noting that at a current density of $20 \mathrm{~mA} \mathrm{~g}^{-1}$ the reversible capacity could be up to $1042 \mathrm{~mA} \mathrm{~h} \mathrm{~g}^{-1}$ after 5 cycles as shown in Fig. 4d, which is higher than other organic materials reported previously. ${ }^{13,16,29}$ The high specific capacity may be ascribed to the high specific surface area and inherent homogeneous microporous structure of PDCzBT, which endow PDCzBT with abundant active sites for Li storage. It can be seen from Fig. $3 c$ that the batteries with PDCzBT based electrodes also exhibit outstanding cycle stability, with a reversible capacity of $404 \mathrm{~mA} \mathrm{~h} \mathrm{~g}^{-1}$ achieved after 100 cycles at a current density of $100 \mathrm{~mA} \mathrm{~g}^{-1}$ and a high coulombic efficiency of $>98 \%$ from the $10^{\text {th }}$ cycle. The PDCzBT electrodes exhibit a specific energy of $224.4 \mathrm{~W} \mathrm{~h} \mathrm{~kg}{ }^{-1}$ based on the mass of the polymer. Even at a high current density of $200 \mathrm{~mA} \mathrm{~g}^{-1}$, the asprepared PDCzBT electrodes deliver a moderate specific capacity of $312 \mathrm{~mA} \mathrm{~h} \mathrm{~g}^{-1}$ after 400 cycles.

For the LIB application, one of the challenging problems is the limited rate performance at high charge-discharge rates. The porous structures have been demonstrated to be beneficial for improving the rate performance of LIBs. The unique structure of PDCzBT with numerous homogeneous micropores is helpful for Li ion diffusion in the polymer chains, thus making PDCzBT suitable as a Li storage material even at high chargedischarge rates. As shown in Fig. S8, $\uparrow$ the $I-V$ curve of PDCzBT is linear, which exhibits Ohmic behavior. The conductivity is
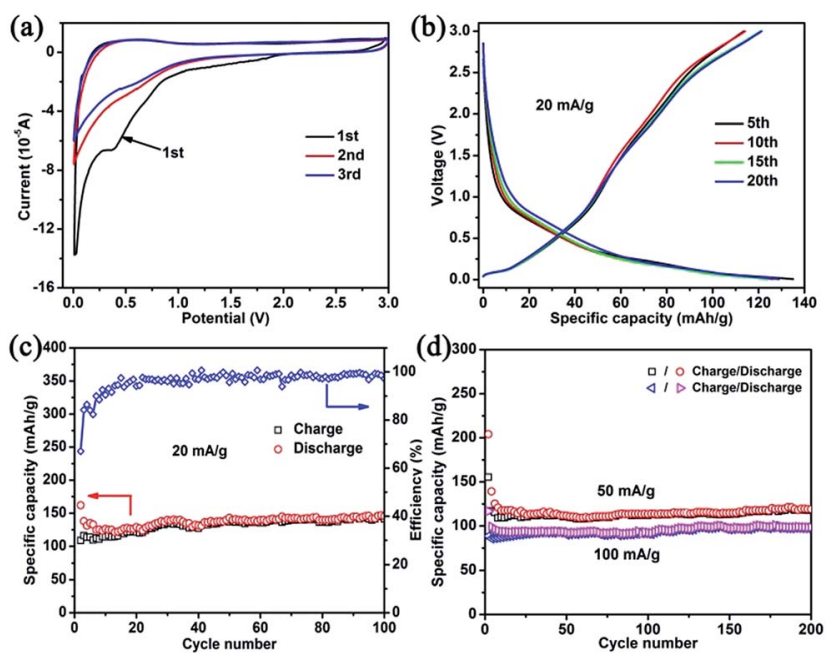

Fig. 4 Electrochemical performance of the PDCzBT electrode for SIBs. (a) Cyclic voltammogram (CV) profiles. (b) Galvanostatic chargedischarge profiles at a current density of $20 \mathrm{~mA} \mathrm{~g}^{-1}$. (c and d) Cycle performance at a current density of 20,50 and $100 \mathrm{~mA} \mathrm{~g}^{-1}$. calculated to be $6.83 \times 10^{-4} \mathrm{~S} \mathrm{~cm}^{-1}$, which is comparable to silicon, and demonstrates that PDCzBT exhibits semiconducting properties and the framework of PDCzBT is helpful for the faster movement of electrons. It can be seen from Fig. $4 \mathrm{~d}$ that PDCzBT demonstrates a superior rate capability. When the current density increased to 500, 1000, and $2000 \mathrm{~mA} \mathrm{~g}^{-1}$, the reversible capacity can still remain at 215, 161 and $117 \mathrm{~mA} \mathrm{~h} \mathrm{~g}{ }^{-1}$, respectively. All these results indicate that PDCzBT is a promising electrode material with high capacity, outstanding rate capability and long cycle life for Li storage devices.

\section{Electrochemical performance of PDCzBT for Na storage}

Recently, SIBs have been recognized as a promising alternative to current LIBs for next generation battery systems and large scale energy storage devices due to the availability of sodium (Na) and the similar chemical properties of $\mathrm{Na}$ and $\mathrm{Li}^{3-5}$ However, many traditional inorganic intercalation materials used in LIBs are usually composed of a 3D rigid network and relatively small tunnel size, which is suitable for diffusion of $\mathrm{Li}^{+}$ but not for $\mathrm{Na}^{+}{ }^{15,36}$ The larger ionic radius of $\mathrm{Na}^{+}$than $\mathrm{Li}^{+}$ results in a larger strain during the insertion/extraction process, which leads to the disintegration of a $3 \mathrm{D}$ rigid network. In contrast, the ion radius has very little effect on the electrochemical performance of n-type organics, mainly due to their flexible framework. Organic materials can accommodate large $\mathrm{Na}$ ions reversibly without much spatial hindrance, thus facilitating fast kinetics to be achieved for $\mathrm{Na}$ ion insertion and extraction reactions. ${ }^{\mathbf{1 4 , 1 5 , 3 7}}$ In addition, organic materials are environmentally friendly, resource sustainable and structurally diverse. Consequently, organic materials might be a good candidate as electrode materials for Na-based energy storage devices.

In this work, we apply PDCzBT as an electrode material for SIBs and investigate its Na storage behavior. It can be seen from Fig. $4 \mathrm{a}$ and $\mathrm{b}$ that the $\mathrm{CV}$ curves and charge-discharge profiles are similar to the curves for lithium storage, only with lower voltage and capacity, which is due to the difference in the thermodynamics and kinetics for the insertion of $\mathrm{Li}$ and $\mathrm{Na}$ ions. Because of the larger ionic radius of $\mathrm{Na}^{+}(102 \mathrm{pm})$ than $\mathrm{Li}^{+}$ (76 pm), the kinetics of $\mathrm{Na}$ insertion and extraction are more sluggish compared to Li. ${ }^{4,38}$ Fig. 4 b displays typical n-doping charge-discharge profiles, and the $\mathrm{Na}$ storage mechanism is also an absorption and insertion process. The insertion process can be based on eqn (2), corresponding to the $\mathrm{Na}^{+}$dopingdedoping into/from the polymers chains. Fig. 4c and d show that the assembled SIBs exhibited a moderate specific capacity and remarkable cycle stability, with a reversible capacity of $145 \mathrm{~mA} \mathrm{~h} \mathrm{~g}{ }^{-1}$ achieved after 100 cycles at a current density of $20 \mathrm{~mA} \mathrm{~g}^{-1}$ and a high coulombic efficiency of $>96 \%$ from the $15^{\text {th }}$ cycle. The difference between $\mathrm{Na}$ storage and Li storage might be attributed to the larger ionic radius and more sluggish kinetics of $\mathrm{Na}^{+}$than $\mathrm{Li}^{+}$, which results in a lower doping and absorbing amount of $\mathrm{Na}^{+}$. The specific explanation is further being studied. At a high current density of 50 and $100 \mathrm{~mA} \mathrm{~h} \mathrm{~g}^{-1}$, the reversible capacity up to 119 and $99 \mathrm{~mA} \mathrm{~h} \mathrm{~g}^{-1}$ (Fig. 4d) could 
be obtained after 200 cycles respectively without any capacity reduction from the $15^{\text {th }}$ cycle, demonstrating the outstanding electrochemical performance of PDCzBT for $\mathrm{Na}$ storage. However, the initial coulombic efficiency is very low at $29.8 \%$ (Fig. S7 $\dagger$ ), and it still needs numerous efforts to study deeply and improve its performance.

$$
\mathrm{PDCzBT}+x \mathrm{Na}^{+}+x \mathrm{e}^{-} \leftrightarrow \mathrm{Na}_{x}{ }^{+} \mathrm{PDCzBT}^{x-}
$$

The excellent electrochemical performance of PDCzBT for $\mathrm{Li}$ and $\mathrm{Na}$ storage can be ascribed to four aspects: (1) conductive CMP frameworks that are conductive to electronic fast transport, (2) abundant redox-active units of its skeletons that provide sufficient energy storing modules, (3) high specific surface areas that endow it with more active sites for storage reactions, and (4) inherent well-developed microporous structures that allow for the fast transport of ions.

\section{Conclusions}

In summary, PDCzBT with a large surface area and uniform microporous structure was facilely prepared by $\mathrm{FeCl}_{3}$ oxidation coupling polymerization and applied as electrode materials for LIBs and SIBs. The high surface area and plentiful redox-active units provide abundant active sites and energy storing modules. The homogeneous microporous structure is beneficial for the fast transport of electrons and ions. The Li/Na storage mechanism of PDCzBT is an absorption and insertion process. The assembled batteries based on PDCzBT electrodes exhibit excellent electrochemical performance for $\mathrm{Li}$ and $\mathrm{Na}$ storage, including high specific capacity, outstanding cycle stability and superior rate performance. These noticeable results demonstrate the enormous potential of CMPs as green, sustainable, flexible and high-performance electrode materials for next generation energy storage devices.

\section{Acknowledgements}

This work was supported by the "100 Talents" program of the Chinese Academy of Sciences, the National Natural Science Foundation of China (21274161), the National Natural Science Foundation of China (21271180) and the National Basic Research 973 Program of China (2011CB935700).

\section{Notes and references}

1 M. Armand and J. M. Tarascon, Nature, 2008, 451, 652-657. 2 V. Etacheri, R. Marom, R. Elazari, G. Salitra and D. Aurbach, Energy Environ. Sci., 2011, 4, 3243-3262.

3 S.-W. Kim, D.-H. Seo, X. Ma, G. Ceder and K. Kang, Adv. Energy Mater., 2012, 2, 710-721.

4 V. Palomares, P. Serras, I. Villaluenga, K. B. Hueso, J. Carretero-Gonzalez and T. Rojo, Energy Environ. Sci., 2012, 5, 5884-5901.

5 M. D. Slater, D. Kim, E. Lee and C. S. Johnson, Adv. Funct. Mater., 2013, 23, 947-958.
6 X. X. Li, F. Y. Cheng, B. Guo and J. Chen, J. Phys. Chem. B, 2005, 109, 14017-14024.

7 M. S. Whittingham, Chem. Rev., 2004, 104, 4271-4301.

8 J. Chen and F. Cheng, Acc. Chem. Res., 2009, 42, 713-723.

9 B. Dunn, H. Kamath and J.-M. Tarascon, Science, 2011, 334, 928-935.

10 X.-P. Gao and H.-X. Yang, Energy Environ. Sci., 2010, 3, 174189.

11 Z. Song and H. Zhou, Energy Environ. Sci., 2013, 6, 2280.

12 X. Feng, Y. Liang, L. Zhi, A. Thomas, D. Wu, I. Lieberwirth, U. Kolb and K. Muellen, Adv. Funct. Mater., 2009, 19, 21252129.

13 M. Armand, S. Grugeon, H. Vezin, S. Laruelle, P. Ribiere, P. Poizot and J. M. Tarascon, Nat. Mater., 2009, 8, 120-125.

14 L. Zhao, J. Zhao, Y.-S. Hu, H. Li, Z. Zhou, M. Armand and L. Chen, Adv. Energy Mater., 2012, 2, 962-965.

15 Y. Park, D. S. Shin, S. H. Woo, N. S. Choi, K. H. Shin, S. M. Oh, K. T. Lee and S. Y. Hong, Adv. Mater., 2012, 24, 3562-3567.

16 L. Zhu, Y. Niu, Y. Cao, A. Lei, X. Ai and H. Yang, Electrochim. Acta, 2012, 78, 27-31.

17 L. M. Zhu, A. W. Lei, Y. L. Cao, X. P. Ai and H. X. Yang, Chem. Commun., 2013, 49, 567-569.

18 T. Suga, S. Sugita, H. Ohshiro, K. Oyaizu and H. Nishide, Adv. Mater., 2011, 23, 751-754.

19 J.-X. Jiang, F. Su, A. Trewin, C. D. Wood, N. L. Campbell, H. Niu, C. Dickinson, A. Y. Ganin, M. J. Rosseinsky, Y. Z. Khimyak and A. I. Cooper, Angew. Chem., Int. Ed., 2007, 46, 8574-8578.

20 Y. Xu, S. Jin, H. Xu, A. Nagai and D. Jiang, Chem. Soc. Rev., 2013, 42, 8012-8031.

21 O. K. Farha, A. M. Spokoyny, B. G. Hauser, Y.-S. Bae, S. E. Brown, R. Q. Snurr, C. A. Mirkin and J. T. Hupp, Chem. Mater., 2009, 21, 3033-3035.

22 W. Lu, D. Yuan, J. Sculley, D. Zhao, R. Krishna and H.-C. Zhou, J. Am. Chem. Soc., 2011, 133, 18126-18129.

23 S. Qiao, Z. Du and R. Yang, J. Mater. Chem. A, 2014, 2, 18771885.

24 Y. He, S. Xiang and B. Chen, J. Am. Chem. Soc., 2011, 133, 14570-14573.

25 P. Kaur, J. T. Hupp and S. T. Nguyen, ACS Catal., 2011, 1, 819-835.

26 Y. Xie, T.-T. Wang, X.-H. Liu, K. Zou and W.-Q. Deng, Nat. Commun., 2013, 4, 1960.

27 Y. Kou, Y. Xu, Z. Guo and D. Jiang, Angew. Chem., Int. Ed., 2011, 50, 8753-8757.

28 F. Xu, X. Chen, Z. Tang, D. Wu, R. Fu and D. Jiang, Chem. Commun., 2014, 50, 4788-4790.

29 K. Sakaushi, E. Hosono, G. Nickerl, H. Zhou, S. Kaskel and J. Eckert, J. Power Sources, 2014, 245, 553-556.

30 K. Sakaushi, E. Hosono, G. Nickerl, T. Gemming, H. Zhou, S. Kaskel and J. Eckert, Nat. Commun., 2013, 4, 1485.

31 Q. Chen, M. Luo, P. Hammershoj, D. Zhou, Y. Han, B. W. Laursen, C.-G. Yan and B.-H. Han, J. Am. Chem. Soc., 2012, 134, 6084-6087.

32 A. R. Paniego, An Quim. Ser Quim. Fisica. Quim. Tecnic., 1989, 85, 386-399. 
33 J. Weber, J. Schmidt, A. Thomas and W. Boehlmann, Langmuir, 2010, 26, 15650-15656.

34 G. Wang, X. Shen, J. Yao and J. Park, Carbon, 2009, 47, 20492053.

35 Y. Yan, B. Hao, D. Wang, G. Chen, E. Markweg, A. Albrecht and P. Schaaf, J. Mater. Chem. A, 2013, 1, 14507-14513.
36 K. M. Abraham, Solid State Ionics, 1982, 7, 199-212.

37 W. Deng, X. Liang, X. Wu, J. Qian, Y. Cao, X. Ai, J. Feng and H. Yang, Sci. Rep., 2013, 3, 2671.

38 C. Zhu, X. Mu, P. A. van Aken, Y. Yu and J. Maier, Angew. Chem., Int. Ed., 2014, 53, 2152-2156. 